\title{
Systems Biology Approaches Towards the Prediction of Prospective Novel Plant System-Derived Products or Services \\ Varoon Vishnu*
}

Department of Biology, University of York, UK

\begin{abstract}
This is a proposed business model for a systems approach in adapting or optimizing plants towards novel products that briefly explains the growth and recent trends in plant systems using three modeling approaches: L-systems, Dynamical-biosystems analysis and cellular automata. The model discusses some of the attempts made by scientists in this field such as model development in Faba bean crops and the auto regulation in nodulation of plants and some other simulated models (ALAMEDA). Its application in horticulture or life style industry is proposed based on the meristem model applied in the roots of Zea mays. L. A grid based approach that effectively simulates and models competition among chosen species of plants on a simulated environment helps in the study of species survival amidst competition from other species, threat from pests, weeds etc. A model for the ecological risk assessment enables the study of impacts of a newly introduced plant variety on the environment and the implications on plants by the environment that helps in decision making and public acceptance. There are attempts in building the virtual cells, by choosing to apply living cell conditions with some basic cell components working effectively, leading to a thrust in the field of systems biology where this would be an application on all kind of cells in any organisms. A plant competition model in an agronomic perspective uses some of the plant models that help in increase of crop yield in agriculture.

Systems biology is an integration of genomics, transcriptomics, proteomics and metabolomics where approaches in identifying the regulatory elements and conserved regulatory motifs have been successfully made leading to a possibility of molecular level study where the input data is scarce and inappropriate beyond which it enables developing hypotheses to decipher poorly understood signaling pathways. A good example of direction and path deduction in plant systems using auxin transport applies best in a well studied business environment enabling a wide range of future perspectives. Some of the novel products or services from plant systems, derived using the systems approaches are briefly discussed. The context of business settings and commercial plausibility is well supported in all attempts of applying the systems biology approach in deriving plant products or services.
\end{abstract}

\section{Introduction}

A systems approach to biology provides a wide and in-depth perception in analyzing complex and dynamic bio-systems. In spite of handling a large amount of data manually, a systems approach provides robust and simple methods of manipulating biological data in order to derive meaningful solutions. A combination of experimental and computational research provides a complete understanding of complex biological systems [1]. Mathematical and computational tools in systems biology enable the development of functional and regulatory networks beneath such bio-systems [2]. With the advent of computer technology and detailed understanding of molecular biology, the use of systems biology has grown tremendously [3] (Figure 1). Drug development and targeting through academy-industry partnerships can employ computer simulation and modeling to analyse biological interactions altered by diseases [2]. Cancer, diabetes and obesity are considered prototypical systems-diseases in which the systems biologists are gathering novel insights [2]. The emergence of high throughput technologies to analyse complex sets of data in both plant and animal models has used computational algorithms [4].

A progress in integrating biophysical, developmental, and genetic information into a useful model will require a system-based approach (Somerville et al.). Kitano [5] defines four key elements in a system: the design principle, system structure, control method, and system dynamics. An attempt to evaluate the current state of knowledge about the polysaccharide components of dicotyledonous plant cell walls in the context of these elements was made by the author. A most appreciable application of systems biology would be creating a cell model of biological system with emphasis on all minute components in it such as signal transduction molecules to provide life in mechanism driven drug discovery [5]. Feedback control and protocols play a major role only in biological science where an effective systems biology approach is more plausible (Marie et al.).

The systems approach in cancers yields numerous solutions at every stage of analysis where identification of key genes involved in cancer using gene regulatory networks is made simpler. Such an approach is used in the identification of the Androgen receptor gene (AR) from the primary and metastatic prostate cancer data and related pathways which conclude that the AR gene and the AR gene pathway are mediators of prostate cancer progression [6]. A multi scale model in analyzing the host-pathogen interaction in $M$. tuberculosis addresses many aspects of immunity and this approach of multi scaling could be applied to a number of tissues such as cardiac and cancer cells [6].

An application of systems approach given by Aldon J.Lusis in Lipidomics, a systems based study on the lipid interactions, states

*Corresponding author: Varoon Vishnu, Department of Biology, University of York, UK, E-mail: varoon2810@gmail.com

Received July 19, 2013; Accepted September 30, 2013; Published October 05 2013

Citation: Vishnu V (2013) Systems Biology Approaches Towards the Prediction of Prospective Novel Plant System-Derived Products or Services. J Comput Sci Syst Biol 6: 286-290. doi:10.4172/jcsb.1000123

Copyright: (c) 2013 Vishnu V. This is an open-access article distributed under the terms of the Creative Commons Attribution License,which permits unrestricted use, distribution, and reproduction in any medium, provided the original author and source are credited. 
that the understanding in the complex interactions of the lipids can unveil the implications of phospholipids in atherosclerosis (Lusis). Putative signaling pathways could be deciphered with the available sparse datasets using state space modeling that hypothesizes poorly interpreted regulatory networks [7]. A vision of personalized medicine can be achieved only through such approaches that well describe the innovative combination of computational and medical knowledge $[8,9]$.

Study on the lipid interactions, states that the understanding in the complex interactions of the lipids can unveil the implications of phospholipids in atherosclerosis (Lusis). Putative signaling pathways could be deciphered with the available sparse datasets using state space modeling that hypothesizes poorly interpreted regulatory networks [7]. A vision of personalized medicine can be achieved only through such approaches that well describe the innovative combination of computational and medical knowledge [7].

\section{Discussion}

The systems approach in complex plant systems yield an in-depth understanding of minute details of plant life. Plant systems are more complicated that the modeling and simulation of the whole system is even more complicated than they appear. Modeling and simulation of individual organs help in the effective usage of systems approach. In 1968, Aristid Lindenmayer introduced formalism for simulating the development of multicellular organisms called L-systems [10]. This system was more widely used in modeling plants. Later, the advent of parametric L-systems which uses the strings of modules associated with parameters became more predominant [11]. It addresses the description of morphogenesis and an emergence of global model and developmental form. These systems provide potentially general solutions than simulations that rely entirely on case studies. We still lack the complete mathematical description of plant systems where a gap between L-systems and the biological problems exists and that needs to be addressed [11] (Figures 2 and 3).

The L-systems approach towards plants is both crucial and a novel approach to model auto-regulation of nodulation both structurally and functionally (Lindenmayer systems) (Han et al.) where the author succeeds in the modeling of branching pattern with regular radial angles between soybean lateral roots using the RULD, a root mapping method
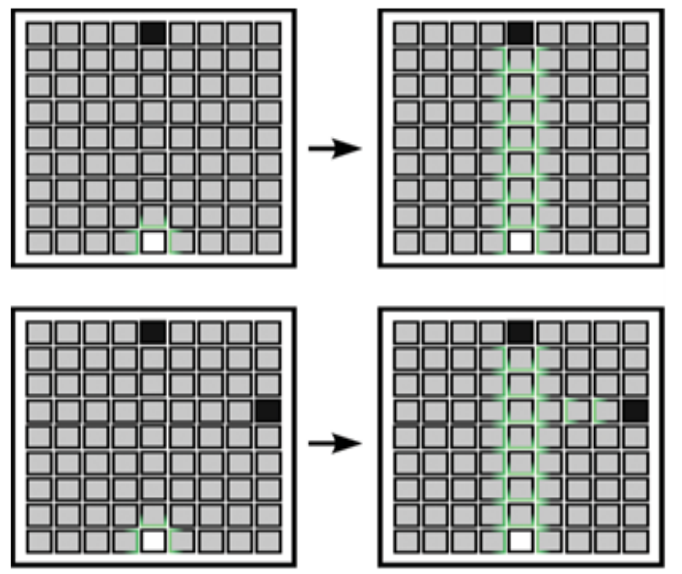

Figure 1: Represents the $2 \mathrm{D}$ square grid of tissues where the white square represents the auxin molecule, black squares represents the auxin source and the green squares represents the Pin localization showing the path of auxin transport [3].

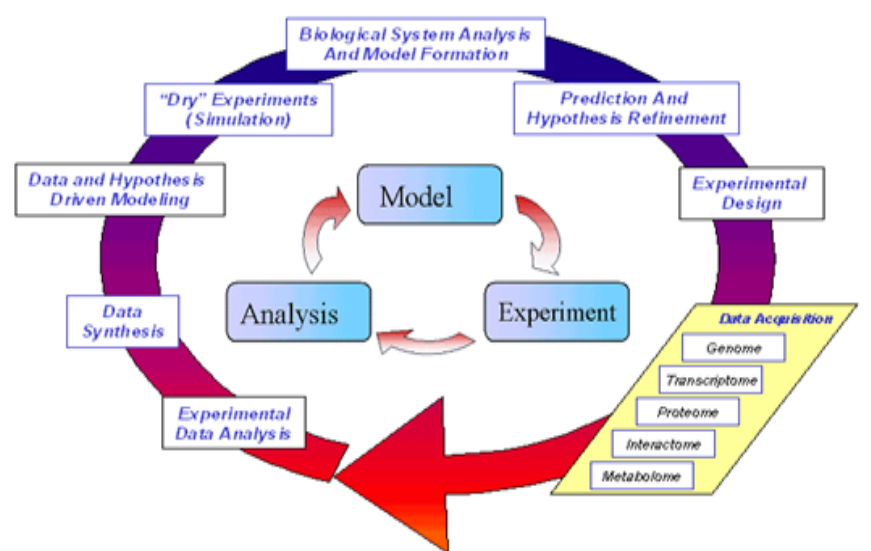

Figure 2: An overall view of a systems biology approach [ http://www.agilent. com/labs/research/sysbio.html as viewed on 20 feb 2011].

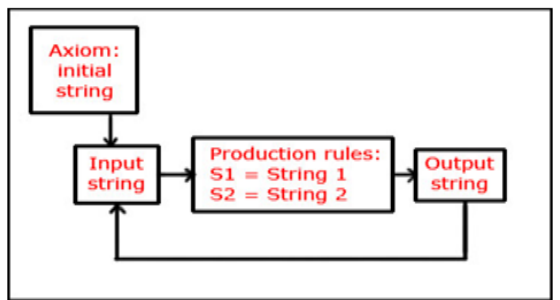

Figure 3: A systematic flow of instructions in L-Systems.[http://www. selcukergen.net/ncca_Isystems_research/lsystems.html]

and comes out with two new algorithms - root elongation algorithm and a synchronization algorithm to coordinate multivariate signaling and developmental processes. However, he fails to consider the second order laterals and mortality of root tips (Han et al.). A same kind of approach was used in the development of a model in Faba bean crops ALAMEDA, a morphological parameterization and verification model. This model includes a combination of advantages of structural and process approaches yielding the number of reproductive nodes that serve as an initial step in developmental processes [12]. The L-systems approach in ALAMEDA provides parametric and environmental sensitive modules considering the parameters like chronological and photo thermal time; however other systems define parametes like rank of organ and phenological stage, shape, size, position and angles [12]. Another theoretical study in the development of meristem in Zea mays, a model used to study the growth of meristem and its manipulation done by the bootstrap L-system algorithm simulates the cellular packet development comprising the cortical portion of the maize root primary meristem [13].

In the field of agronomy, L-system is used in determining crop competition with respect to plant spacing, where it applies to crop-weed competition in yield assessments [14] and a similar model in assessing the plant-pest interactions enables the simulation studies of insect used for weed control and impacts on crops [15]. One of the concepts of systems biology is "The Dynamical systems analysis", which involves the evolution of systems over time using attractors. Attractors are a set towards a dynamical system evolving over time. An attempt to identify interacting regulators and conserved regulations in Populus leaves using a systems biology model was successfully achieved. Quantified Transcriptional profiles across 465 samples of Populus in various 
experiments were used in deriving representational transcriptional modules containing co-regulatory promoters in co-expressed genes in these samples. The author inferred a number of regulatory elements involved in the leaf development under an assumption that common sequence motifs on promoters and similar expression profiles would be observed in genes that are regulated by the same transcription factors. The approach seemed promising and robust in new and nonobvious data, extracting the conserved regulatory elements in different conditions within and between Populus and in Arabidopsis (Street). Such approaches could be applied in plants that are medicinally important to understand and enhance the treating ability of plants.

A morphogenetic model that best explains the aperture formation during meiosis stage considering nuclei as attractors leads to the partitioning of the cell into apertures of pollens. The author attempts to explain the duplication of an aperture of a three aperturate pollen grain leads to formation of four apertures and heterogeneous tetrads [16]. The algorithm builds a vector field inside a ball that represents the cells centering the nuclei. The space in the ball is divided to make domains in each attractor where each attractor has particles that cannot move to other attractors but can remain moving in the same attractor giving rise to arms and the points were the arms meet are considered to indicate the position of aperture [16]. A more robust approach is the model of plant competition in an agronomic perspective that considers the plant competition fundamentals, plant-weed interaction and the dynamics of weeds in crops. Crop-weed competition is widely analyzed in the estimation of crop yield with respect to the weed influence on the crop in the field and a similar kind of approach [14] by Streibig in 1989, in the estimation of crop yield with respect to the herbicide use on crops. One of the main advantages of such an approach is that they are applicable to insufficient and uncertain data [17]

Decision making aids such as WEEDSIM [18] and WEEDCAM [19] provides estimates or outcomes based on the initial parameters of seed and weed population. Analysis of regulatory networks involves more time and labour which is now saved by the system approach in all aspects of analysis. Some of the methods to critically assess regulatory networks were formulated by Marbach et al. [20] (Figure 4, Table 1).

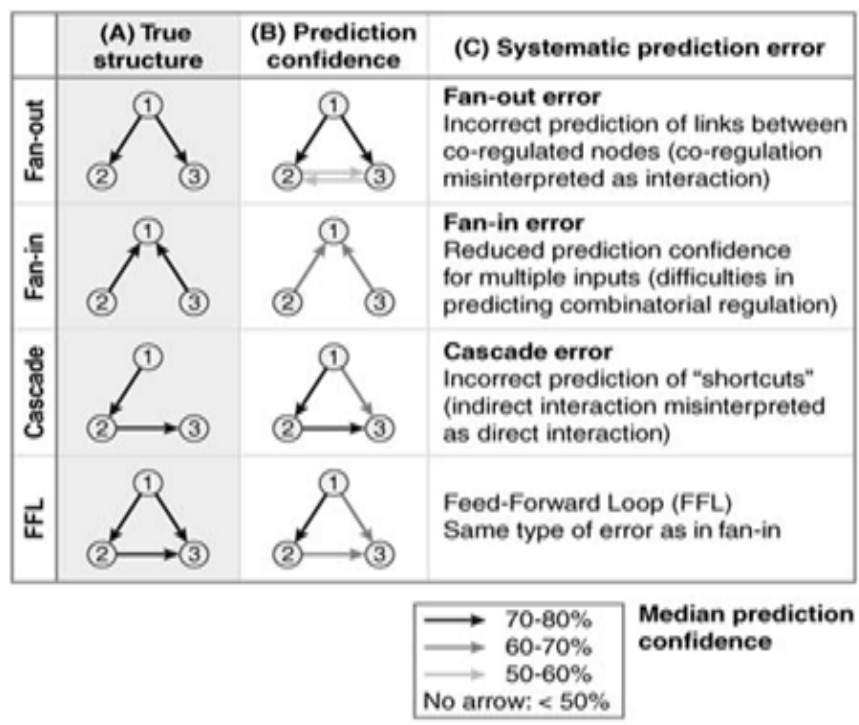

Figure 4: Three possible Systematic errors in the prediction of network motifs where the darkness of the links predicts the median prediction confidence [20].
An assessment on 29 gene regulatory networks inferring methods was performed by independent teams that employed them. A method by Yip and co-workers in 2010 proved to be the best amongst the methods that were used. Besides other methods like correlation based methods, Bayesian network prediction, information theoretic methods and methods based on dynamical models have also produced promising results and the author came out with three possible errors in systematic prediction of network motifs such as fan out error, fan in error and cascade error [20].

These errors also arise due to the inaccurate assumption that considerably affects the performance of inferring methods [20].

A cellular automaton is a collection of cells on a grid of specified shape that evolves through a number of discrete time steps according to a set of rules based on the states of neighboring cells followed by iteratively performing the rules for a particular time scale. Von Neumann was one of the first people to consider such a model, and incorporated a cellular model into his work. A best computer simulation by Garnet and co-workers in 2010, attempted the simulation of auxin transport in plants where auxin plays a crucial role in the growth of the plant system. The auxin transport canalization simulation in a $2 \mathrm{D}$ grid of square cells representing tissues and cells acting as actin sources are uniquely identified using pin localization to describe the path of auxin movement where it is drained by surrounding cells tissues.

The approach of tracking the auxin transport could be applied in tracking certain biologically important molecules in any living system incorporating computational constraints. As a part of the systems approach, L-system in plants has a wider scope and applies to all plant-based industries that derive plant products or services, directly or indirectly. A grid based simulation model on the sexual and vegetative reproduction of Heracium pilosella under the influence of a competition from a clonal grass species in which each cell in the grid can have host of rosette of pilosella or grass vegetation or could be empty in the time scale of a year. In this case, there is no equal importance given to the biological systems that are under study and not all computationally successful models are biologically successful. The author fails to consider the condition of intra-species competition where it is more important to have the fittest individuals leading to a good population and a high rate of species survival. In the presence of inter-species competition and a shortage of space, a mixed strategy of clonal growth and reproduction by seeds in Pilosella is crucial.

A recent application is in the ecological risk assessment of genetically modified crops where a simple subsystem with four species such as crop, target pest, non-target pest and enemy insects are considered and by using 'penna model' the individual survival state is determined [8]. A parameter to study starvation is also used and the grid becomes empty if the individual dies. A cellular automaton thus allows the implementation of all possible parameters that is needed in building a biological model. The results would be used in the decision making process and in public acceptance [8]. A novel approach that estimates parameters in the model of spread of invasive species in geographical information system (GIS) using the cellular automata where the spatial growth pattern is studied using the tested parameters such as vegetative dynamics and combines the spatial data and the presentation ability of geographical information system (GIS) (Cole, 1999). A discrete event modeling and simulation could be done using the open software CELLDEVS [http://sourceforge.net/projects/cdpptoolkit/ viewed on $20 \mathrm{feb}$ 2011]. Resources on plant growth models could be found in the plant systems biology modeling database (PlasMo)[http://www.plasmo. ed.ac.uk/plasmo/pages/show_page.shtml;jsessionid=4295FCE7F055 
Citation: Vishnu V (2013) Systems Biology Approaches Towards the Prediction of Prospective Novel Plant System-Derived Products or Services. J Comput Sci Syst Biol 6: 286-290. doi:10.4172/jcsb.1000123

\begin{tabular}{|c|c|c|c|c|c|}
\hline Team Name & Members & Affiliation & Rank & Type of method & References \\
\hline B Team & $\begin{array}{l}\text { Kevin Y.Yip, Roger P. Alexander, Koon-Kiu } \\
\text { Yan and Mark Gerstein }\end{array}$ & Yale University, USA & 1st on all sizes & Statistical Method & [2] \\
\hline USMtec347 & Peng Li and Chaoyang Zhang & University of Mississippi, USA & $\begin{array}{l}\text { 2nd on size 10, } \\
\text { 2nd on size } 50\end{array}$ & Bayesian network & - \\
\hline Bonneau & $\begin{array}{l}\text { Aviv Madar, Alex Greenfield, Eric Vanden- } \\
\text { Eijnden, and Richard Bonneau }\end{array}$ & New York University, USA & 2nd on size 100 & Dynamical Model & [3-5] \\
\hline Intigern & Xuebing Wu, Feng Zeng, and Rui Jiang & Tsinghua University, China & $\begin{array}{l}\text { 3rd on size } 10 \\
\text { 3rd on size } 100\end{array}$ & Statistical Method & - \\
\hline Zuma & Tarmo Aijo and Lahdesmaki & Tampere University of Technology, Finland & 3rd on size 50 & Dynamical Model & [6] \\
\hline
\end{tabular}

Table 1: Shows the top five best performing methods in inferring the network motifs in regulatory networks. In contrast, these represented errors in inferring the network motifs in regulatory networks may vary from networks to networks, so the efficiency of the methods should be cautiously studied based on the complexity of networks under study [20].

18DD41AA8C74F7D3949F?name=home as viewed on 20 feb 2011]. L-studio is a context sensitive L-system modeling stands alone, free software [http://algorithmicbotany.org/lstudio/whatis.html as viewed on 20 feb 2011].

Thus, the application of systems biology over the complex biosystems is more widely accepted and all the above implications in any organism especially in plant systems ensures success at any cost as systems biology involves deriving hypothesis from meager amount of unrelated data. So, it would be more effective and fruitful if the company takes in hand, a systems approach to adapt or optimize plants towards novel products that will benefit clients.

\section{Conclusion}

\section{Three Prospective novel plant system-derived products or services:}

Some of the novel plant derived products or services could be:

1. A virtual cell environment could be created using the parameterized L-system where the user can define the components of the cell and study the whole system where the user can expect the system to produce the desired outcome. A similar approach could be used in developing plant derived medicines where a plant system that secretes some proteins or secondary metabolites which will be used as medicines by optimizing the systems parameter at the input stage of a simulation process such that a particular protein or a direct product or by-product of any described process is obtained in a required quantity. A virtual cell is build according to the end-user's need. This kind of approach will also lead to a deep understanding of biology under the plant system and effective manipulation of such parameters in achieving desired results. Changes to any basic biosystem parameter will definitely earn a massive change at the phenotype level. Desired results such as enhanced secretion of certain proteins or secondary metabolites or decrease the secretion of certain products that is a result of any biological process that may bring down the negative effects of certain molecules or process. Such an approach will help in predicting the biological systems behavior when certain compounds are introduced or when the system is perturbed. We can manipulate plant genomes to observe gene expression and phenotype. This kind of approach is possible as there models to support the development of a virtual cell with very less number of components and this could be made possible in any commercial setting.

2. As an extension to the attempt by [13] that best models the meristem development applied to the primary roots of Zea mays, a similar kind of attempt could be made in the field of horticulture and life style. In the above approach the author builds a theoretical model in the meristem development where a complete control over this process is in the hands of the alogorithm, inhibition of the development process may lead to a poor growth of meristem where a new type of "bonsai" could be developed for life style companies. Manipulation of genes that govern color, shape and yield of flowers, leaves and fruits, would yield some bright and innovative man made wonders that serve as ornamental plants that decorate houses, gardens and offices. Such an approach would definitely fit in any commercial setting but it would take more time to be implemented where this would have some bio-ethics issues to be dealt with as this might sound similar to the genetic engineering.

3. The need of the hour plant-derived product is the "Biofuels", from Jatropa curcus where the seeds can be harvested and used in the extraction of bio-diesel. A systems approach in this would be corroborated, as the modeling and simulation of a Jatropa plant system that enables the deeper understanding of the biology in it and a manipulation at the cell parameter level would yield a big quantity and high quality of biodiesels. A simple cellular automata simulation would help us in the study on the risk assessment of the extracted bio-fuel on the environment that helps in decision making and public acceptance.

This kind of approach towards a highly needed technology that would serve the whole world's need has got more investment and returns and is commercially promising. Thus, a systems approach in any kind of plant systems would lead to an in-depth understanding of the hidden nature's concepts and formulae that will open gates for the Novel plant-based products and services for the humanity.

\section{References}

1. Kitano H (2002) Computational systems biology. Nature 420: 206-210.

2. Auffray C, Chen Z, Hood L (2009) Systems medicine: the future of medical genomics and healthcare. Genome Med 1: 2.

3. Garnett P, Steinacher A, Stepney S, Clayton R, Leyser O (2010) Computer simulation: the imaginary friend of auxin transport biology. Bioessays 32: 828835 .

4. Kharabian A (2010) An efficient computational method for screening functional SNPs in plants. J Theor Biol 265: 55-62.

5. Kitano H (2002) Systems biology: a brief overview. Science 295: 1662-1664.

6. Ergün A, Lawrence CA, Kohanski MA, Brennan TA, Collins JJ (2007) A network biology approach to prostate cancer. Mol Syst Biol 3: 82 . 
Citation: Vishnu V (2013) Systems Biology Approaches Towards the Prediction of Prospective Novel Plant System-Derived Products or Services. J Comput Sci Syst Biol 6: 286-290. doi:10.4172/jcsb.1000123

7. Yip KY, Alexander RP, Yan KK, Gerstein M (2010) Improved reconstruction of in silico gene regulatory networks by integrating knockout and perturbation data. PLoS One 5: e8121.

8. Yang J, Wang ZR, Yang DL, Yang Q, Yan J, et al. (2009) Ecological risk assessment of genetically modified crops based on cellular automata modeling. Biotechnol Adv 27: 1132-1136.

9. van der Greef J, Martin S, Juhasz P, Adourian A, Plasterer T, et al. (2007) The art and practice of systems biology in medicine: mapping patterns of relationships. J Proteome Res 6: 1540-1559.

10. Lindenmayer (1968) Mathematical models for cellular interaction in development, Parts I and II. Journal of Theoretical Biology 18: 280-315.

11. Prusinkiewicz P, Rolland-Lagan AG (2006) Modeling plant morphogenesis Curr Opin Plant Biol 9: 83-88.

12. Ruiz-Ramos M, Mínguez MI (2006) ALAMEDA, a structural-functional model for faba bean crops: morphological parameterization and verification. Ann Bot 97: $377-388$

13. Luck J, Barlow PW, Luck HB (1997) An Automata-theoretical Model of Meristem Development as Applied to the Primary Root of Zea mays L. Annals of botany 79: $375-389$
14. Park SE, Benjamin LR, Watkinson AR (2003) The theory and application of plant competition models: an agronomic perspective. Ann Bot 92: 741-748.

15. Prusinkiewicz P, Allen M, Escobar-Gutierrez A, DeJong TM (2007) Numerica methods for transport-resistance source-sink allocation models. FunctionalStructural Plant Modelling in Crop Production 22.

16. Ressayre A, Godelle B, Mignot A, Gouyon PH (1998) A Morphogenetic Mode Accounting for Pollen Aperture Pattern in Flowering Plants. J Theor Biol 193 321-334.

17. Auld BA, Tris dell (1987) Economic thresholds and response to uncertainty in weed control. Agricultural Systems 25: 219-227.

18. Swinton AM, Robert KP (1994) A bio-economic model for weed management in corn and soybean. Agricultural Systems 44: 313-335.

19. Lybecker DW, Edward ES, Robert PK (1991) Weed management decisions in corn based on bio-economic modeling. Weed 39: 124-129.

20. Marbach D, Prill RJ, Schaffter T, Mattiussi C, Floreano D (2010) Revealing strengths and weaknesses of methods for gene network inference. PNAS 107: 14. 